\title{
Reclassification of Clostridium proteoclasticum as Butyrivibrio proteoclasticus comb. nov., a butyrate- producing ruminal bacterium
}

Correspondence

Christina D. Moon

christina.moon@agresearch.co.nz

\author{
Christina D. Moon, ${ }^{1}$ Diana M. Pacheco, ${ }^{1}$ William J. Kelly, ${ }^{1}$ \\ Sinead C. Leahy, ${ }^{1}$ Dong Li, ${ }^{1}$ Jan Kopečný ${ }^{2}$ and Graeme T. Attwood ${ }^{1}$
${ }^{1}$ Food, Metabolism and Microbiology Section, AgResearch Ltd, Grasslands Research Centre, Tennent Drive, Private Bag 11008, Palmerston North, New Zealand
${ }^{2}$ Institute of Animal Physiology and Genetics, Czech Academy of Sciences, Vídeňská 1083, 142 20, Prague 4, Krč, Czech Republic

\begin{abstract}
It is proposed that Clostridium proteoclasticum be reclassified as Butyrivibrio proteoclasticus comb. nov. on the basis of phylogenetic position, DNA $\mathrm{G}+\mathrm{C}$ content and physiological traits. Phylogenetic analyses based on 16S rRNA gene sequences from an extensive range of taxa within clostridial rRNA subcluster XIVa grouped C. proteoclasticum together with isolates of the genus Butyrivibrio, though this species was genetically distinct from the extant Butyrivibrio species examined. The DNA $\mathrm{G}+\mathrm{C}$ content of $\mathrm{C}$. proteoclasticum was originally erroneously reported as $28 \mathrm{~mol} \%$. However the genome sequence of the type strain of C. proteoclasticum, strain $\mathrm{B}^{3} 16^{\top}$, and HPLC analysis estimate the DNA G+C content as $40 \mathrm{~mol} \%$, which is within the range reported for strains of Butyrivibrio. C. proteoclasticum was distinguishable from other species of the genus Butyrivibrio as the $16 \mathrm{~S}$ rRNA gene from strain B316 ${ }^{\top}$ shared less than $97 \%$ sequence similarity with sequences from the type strains of Butyrivibrio species. C.

proteoclasticum was also able to convert linoleic acid to stearic acid, in contrast to other species of Butyrivibrio. Physiological characteristics, including carbon source utilization, volatile fatty acid production and proteinase activities, were assessed for a panel of representative strains of the genera Butyrivibrio and Pseudobutyrivibrio and C. proteoclasticum. These data, together with the phylogenetic analyses, support the reclassification of Clostridium proteoclasticum as a separate species within the genus Butyrivibrio, Butyrivibrio proteoclasticus comb. nov. (type strain B316 ${ }^{\top}=$ ATCC $51982^{\top}=$ DSM $14932^{\top}$ ).
\end{abstract}

The original principal characteristics of members of the genus Butyrivibrio (family Lachnospiraceae) (Bryant \& Small, 1956; Bryant, 1986) are that they possess curved, strictly anaerobic, non-spore-forming, rod-shaped cells that are generally motile by means of one or more polar to subpolar flagella. They possess an atypical Gram-positive ultrastructure, although stain Gram-negative (Bryant, 1986), and have a fermentative metabolism with butyrate

\footnotetext{
Abbreviation: VFA, volatile fatty acid.

The GenBank/EMBL/DDBJ accession numbers for the 16S rRNA gene sequences of Butyrivibrio fibrisolvens $\mathrm{C} 219 \mathrm{a}$ and Pseudobutyrivibrio ruminis CF1b are EU346756 and EU346757, respectively.

Supplementary tables detailing the strains used in this study and the 16S rRNA gene sequence similarity for strains of the genera Butyrivibrio and Pseudobutyrivibrio and a supplementary figure showing a multisequence alignment of partial 16S rRNA genes from strains of the genera Butyrivibrio, Pseudobutyrivibrio, Clostridium, Ruminococcus and Bacillus generated using CLUSTAL W are available with the online version of this paper.
}

as the main product. Strains of the genus Butyrivibrio are commonly isolated from the rumen and the mammalian gastrointestinal tract and have a DNA G + C content of 36$42 \mathrm{~mol} \%$ (Bryant, 1986). Phylogenetic analyses have revealed that the genus Butyrivibrio is a diverse polyphyletic assemblage (Willems et al., 1996) for which three species: Butyrivibrio fibrisolvens (the type species), Butyrivibrio crossotus and Butyrivibrio hungatei are currently recognized (Bryant \& Small, 1956; Kopečný et al., 2003; Moore et al., 1976). Clostridium proteoclasticum, exemplified by the type strain $\mathrm{B} 316^{\mathrm{T}}\left(=\mathrm{ATCC} 51982^{\mathrm{T}}=\mathrm{DSM} 14932^{\mathrm{T}}\right)$, was first proposed in 1996 (Attwood et al., 1996) to describe a strictly anaerobic Gram-positive bacterium with straight to slightly curved rod-shaped cells bearing a single subpolar flagellum, isolated from the rumen of a New Zealand cow (Attwood \& Reilly, 1995). Accordingly, this highly proteolytic bacterium was originally described as Butyrivibrio-like (Attwood \& Reilly, 1995). However, its apparently much lower DNA G $+\mathrm{C}$ content (originally 
reported as $28 \mathrm{~mol} \%$, which is typical of the Clostridium type species, Clostridium butyricum), lack of motility and lack of dextrin utilization (Attwood et al., 1996) distinguished strain $\mathrm{B} 316^{\mathrm{T}}$ from B. fibrisolvens. Furthermore, phenotypic characterization and phylogenetic analyses of $16 \mathrm{~S}$ rRNA genes showed that strain $\mathrm{B} 316^{\mathrm{T}}$ was most closely related to Clostridium aminophilum within clostridial subcluster XIVa, a strongly supported cluster defined on the basis of phylogenetic analysis of $16 \mathrm{~S}$ rRNA genes from an extensive range of clostridial strains (Collins et al., 1994). The same analysis demonstrated that the genus Clostridium is highly diverse, with strains often intermixed with members of many different genera (Collins et al., 1994). However, it is generally accepted that the genus Clostridium should be retained only for organisms belonging to cluster I, which contains the type species (Collins et al., 1994). It was initially proposed that strain $\mathrm{B} 316^{\mathrm{T}}$ be assigned to a novel species of the genus Clostridium, $C$. proteoclasticum, with a view that classification was likely to change upon review of clostridial subcluster XIVa (Attwood et al., 1996).

Since the original description of C. proteoclasticum, a body of evidence to support its reclassification to the genus Butyrivibrio has arisen. It has been found that the DNA $\mathrm{G}+\mathrm{C}$ content originally reported for strain $\mathrm{B} 316^{\mathrm{T}}$, $28 \mathrm{~mol} \%$ (Attwood et al., 1996), was erroneous and recent estimates now place the value in the range of that characteristic for species of the genus Butyrivibrio. Furthermore, phylogenetic analyses involving 16S rRNA gene sequences from a wider range of taxa that fit into clostridial subcluster XIVa, including those from the genera Butyrivibrio and Pseudobutyrivibrio, have been performed (Forster et al., 1996; Kopečný et al., 2001, 2003; Wallace et al., 2006; Willems et al., 1996). Such analyses show that $C$. proteoclasticum sequences cluster closely with sequences of the genus Butyrivibrio, where it is most closely related to $B$. hungatei (Kopečný et al., 2003; Wallace et al., 2006). In addition, PCR primers designed specifically to amplify $16 \mathrm{~S}$ rRNA gene sequences from species of the genus Butyrivibrio (Mrázek \& Kopečný, 2001) were able to amplify sequences from $C$. proteoclasticum isolates (Kopečný et al., 2003). Despite its close relationship with $B$. hungatei, C. proteoclasticum can be distinguished from this species by its ability to form stearic acid from linoleic acid (Paillard et al., 2007; Wallace et al., 2006), a feature that has not been observed in other strains of the genera Butyrivibrio or Pseudobutyrivibrio (Paillard et al., 2007).

To support the reclassification of $C$. proteoclasticum to $B$. proteoclasticus comb. nov., we present phylogenetic analyses and a phenotypic characterization of strains of the genera Butyrivibrio and Pseudobutyrivibrio (listed in Supplementary Table S1 in IJSEM Online), including the type strains B. crossotus DSM $2876^{\mathrm{T}}, B$. fibrisolvens ATCC $19171^{\mathrm{T}}$, B. hungatei JK $615^{\mathrm{T}}$, Pseudobutyrivibrio ruminis DSM $9787^{\mathrm{T}}$ and Pseudobutyrivibrio xylanivorans $\mathrm{Mz} 5^{\mathrm{T}}$. Hereafter, we refer to C. proteoclasticum as B. proteoclasticus.
The evolutionary positions of strains of $B$. proteoclasticus [B316 ${ }^{\mathrm{T}}, \mathrm{UC142}, \mathrm{H} 17 \mathrm{c}(\mathrm{SA})$, DSM 10301 and DSM 10304] relative to other strains of the genera Butyrivibrio, Pseudobutyrivibrio, Clostridium and Ruminococcus from clostridial cluster I and subcluster XIVa (Collins et al., 1994) were inferred by $16 \mathrm{~S}$ rRNA gene sequence-based phylogenetic analyses. Near full-length $16 \mathrm{~S}$ rRNA gene sequences ( $>1400 \mathrm{bp}$ ) were obtained by PCR amplification and automated sequencing or were retrieved from GenBank and the Ribosomal Database II databases (Cole et al., 2007). Sequence alignment and phylogenetic analyses were implemented in MEGA4 (Tamura et al., 2007). A multi-sequence alignment was generated using CLUSTAL $\mathrm{W}$ and corrected manually for alignment ambiguities (see Supplementary Fig. S1 in IJSEM Online). A phylogenetic tree was constructed using the neighbour-joining method (Saitou \& Nei, 1987) with the Kimura two-parameter model (transition to transversion ratio $=2.0$ ) for distance estimation (Kimura, 1980). Bootstrap values (Felsenstein, 1985) were determined from 10000 replicates. The resulting phylogenetic tree (Fig. 1) clearly shows that $B$. proteoclasticus sequences are located in clostridial subcluster XIVa (rather than cluster I), and group together in a well-supported clade ( $88 \%$ bootstrap support), which is closely related to clades containing $B$. hungatei strains and Butyrivibrio strains NCDO 2222 and NCDO 2434. Despite this close relationship, the 16S rRNA gene sequence similarity between the type strains of $B$. proteoclasticus and B. hungatei is $95.7 \%$ (see Supplementary Table S2 in IJSEM Online), which is below the cut off value (97\%) that is generally accepted for two strains to be considered members of the same species (Stackebrandt \& Goebel, 1994; Staley, 2006). Interestingly, strains NCDO 2222 and NCDO 2434 were also considered to be isolates of $C$. proteoclasticum on the basis of their ability to convert linoleic acid to stearic acid (Paillard et al., 2007), and NCDO 2222 was earlier considered to be a B. hungatei strain (Kopečný et al., 2003). However, the distinct position of these two strains within the 16S rRNA gene phylogeny (Fig. 1 and Wallace et al., 2006) and <97\% 16S rRNA gene sequence similarity to other type strains of the genus Butyrivibrio (see Supplementary Table S2) suggests that strains NCDO 2222 and NCDO 2434 are neither representatives of $B$. proteoclasticus nor B. hungatei, and possibly represent a novel species of the genus Butyrivibrio (and thus are referred to only as Butyrivibrio strains in this study).

Estimation of DNA $\mathrm{G}+\mathrm{C}$ content was determined by enzymic hydrolysis of total genomic DNA, followed by HPLC analyses as previously described (Kopečný et al., 2003). For each strain, the mean $G+C$ content value was determined from two replicate experiments. Values for $B$. proteoclasticus strains $\left(\mathrm{B} 316^{\mathrm{T}}, 40.04 \mathrm{~mol} \%\right.$; DSM 10301, $40.09 \mathrm{~mol} \%$; DSM 10304, $39.72 \mathrm{~mol} \%$; DSM 10305, $42.67 \mathrm{~mol} \%$; JK $669,39.79 \mathrm{~mol} \%$, and UC 142, $41.06 \mathrm{~mol} \%$ ) were within the general range specified for species of the genus Butyrivibrio, in contrast to the original 


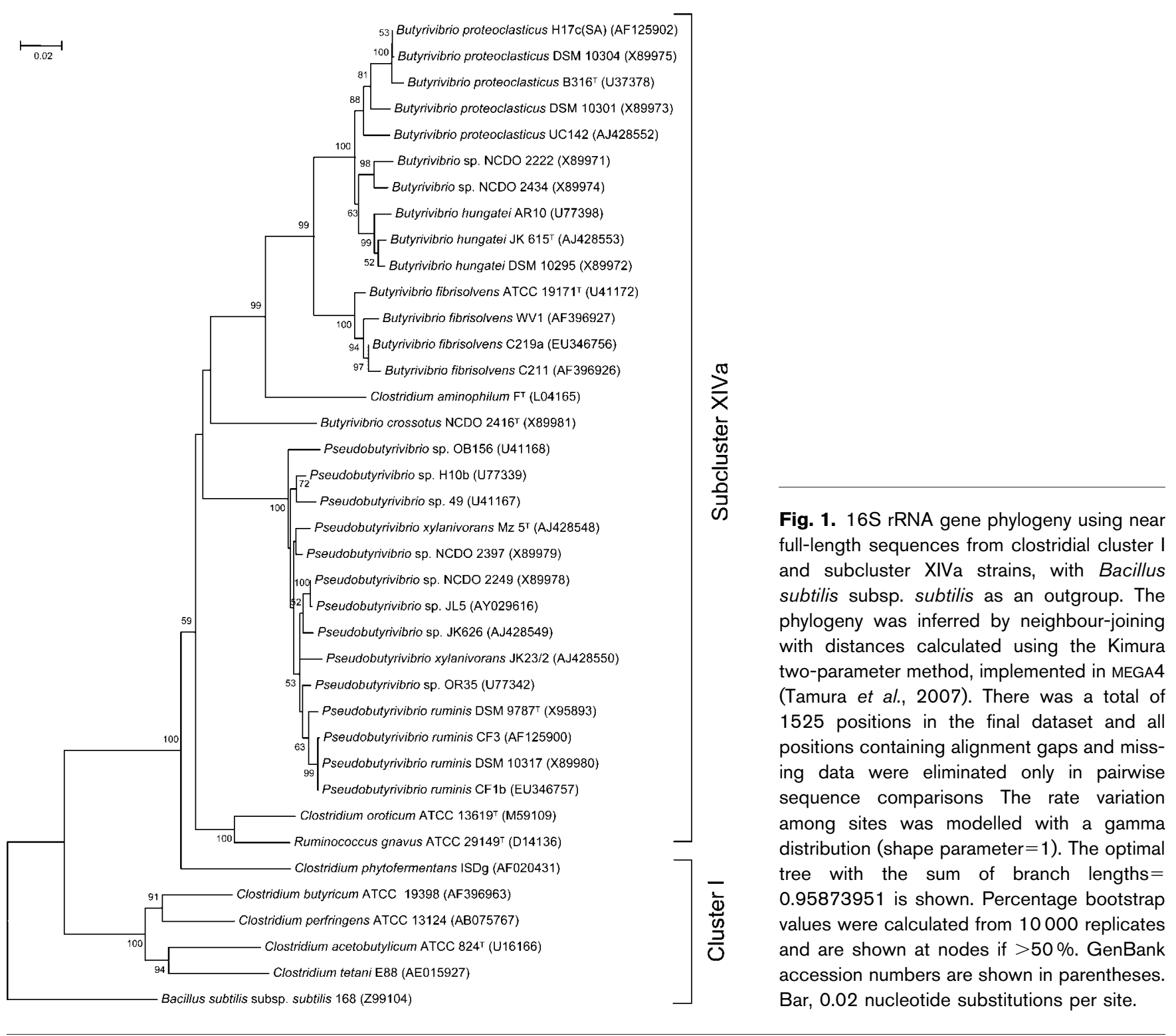

reported $\mathrm{G}+\mathrm{C}$ value for strain $\mathrm{B} 316^{\mathrm{T}}$ of $28 \mathrm{~mol} \%$ (Attwood et al., 1996). These values were further supported by the recent availability of the complete genome sequence of strain $\mathrm{B} 316^{\mathrm{T}}$, where the $\mathrm{G}+\mathrm{C}$ content of the main chromosome is $40.21 \mathrm{~mol} \%$ and an estimated value for the whole genome (considering one copy of each extrachromosomal element) is $40.01 \mathrm{~mol} \%$ (W. J. Kelly, E. Altermann, S. C. Leahy, C. J. Yeoman, C. D. Moon and G. T. Attwood, unpublished information).

A panel of fifteen strains of the genera Butyrivibrio and Pseudobutyrivibrio was assessed for carbon source utilization, proteinase activity and volatile fatty acid (VFA) production. The ability to utilize 30 different carbon sources was tested by growth in DSM Medium 330 broth (modified by omission of all carbohydrates and containing four times the specified amount of yeast extract and double the specified volume of mineral solution), with addition of each specific carbon source at $0.5 \% \mathrm{w} / \mathrm{v}$ final concentration. Cultures were inoculated in duplicate and grown under anaerobic conditions overnight at $39{ }^{\circ} \mathrm{C}$, with results shown in Table 1. Additionally, the substrate utilization abilities of the B. proteoclasticus strains DSM 10301, DSM 10304, DSM 10305, JK 669, JK 722, UC 142 and X2D62 were tested in triplicate using API 20A test kits (bioMérieux; Table 1). In comparison to its closest relatives, B. hungatei and Butyrivibrio sp. NCDO 2222, B. proteoclasticus differed from $B$. hungatei in terms of its ability to utilize amygdalin, glycogen, mannose and melezitose. B. proteoclasticus also differed from strain NCDO 2222 in its ability to utilize fructose, inulin, mannose, melezitose, rhamnose and trehalose (Table 1). In contrast to the original description, strain $\mathrm{B} 316^{\mathrm{T}}$ grew on dextrin and trehalose, but growth on mannose and rhamnose was not observed as originally reported 
Table 1. Carbon source utilization of strains of the genera Butyrivibrio and Pseudobutyrivibrio

Taxa: $1, B$. proteoclasticus strain $\mathrm{B} 316^{\mathrm{T}}$ (or where indicated by asterisk, strains B316 ${ }^{\mathrm{T}}$, DSM 10301, DSM 10304, DSM 10305, JK 669, JK 722, UC 142 and X2D62 analysed); 2, Butyrivibrio sp. NCDO 2222; 3, B. hungatei strains JK $615^{\mathrm{T}}$, AR10 and DSM 10295; 4, B. fibrisolvens strains D1 ${ }^{\mathrm{T}}, \mathrm{C} 211, \mathrm{C} 219 \mathrm{a}$ and WV1; 5, B. crossotus DSM 2876 $;$;,$P$. ruminis strains DSM 9787 ${ }^{\mathrm{T}}$, DSM 10317, CF1b and CF3; 7, $P$. xylanivorans $\mathrm{Mz} 5^{\mathrm{T}}$. Results are expressed as a percentage of positive results.

\begin{tabular}{|lcrrrrrr|}
\hline Substrate & $\mathbf{1}$ & $\mathbf{2}$ & $\mathbf{3}$ & $\mathbf{4}$ & $\mathbf{5}$ & $\mathbf{6}$ & $\mathbf{7}$ \\
\hline Aesculin & 100 & 100 & 100 & 100 & 0 & 100 & 100 \\
Amygdalin & 100 & 100 & 0 & 25 & 0 & 100 & 100 \\
Arabinose & $75^{*}$ & 100 & 100 & 75 & 0 & 100 & 100 \\
Cellobiose & 100 & 100 & 67 & 100 & 0 & 100 & 100 \\
Dextrin & 100 & 100 & 67 & 100 & 100 & 100 & 100 \\
Fructose & 100 & 0 & 100 & 100 & 0 & 100 & 100 \\
Galactose & 100 & 100 & 67 & 100 & 0 & 100 & 100 \\
Glucose & 100 & 100 & 100 & 100 & 0 & 100 & 100 \\
Glycerol & $0^{*}$ & 0 & 0 & 0 & 0 & 0 & 0 \\
Glycogen & 100 & 100 & 0 & 25 & 100 & 75 & 100 \\
Inositol & 0 & 0 & 0 & 0 & 0 & 0 & 0 \\
Inulin & 100 & 0 & 100 & 100 & 0 & 100 & 100 \\
Lactose & $100^{*}$ & 100 & 67 & 100 & 100 & 100 & 100 \\
Maltose & 100 & 100 & 67 & 100 & 100 & 100 & 100 \\
Mannitol & $25^{*}$ & 0 & 0 & 0 & 0 & 0 & 0 \\
Mannose & $88^{*}$ & 0 & 0 & 50 & 0 & 50 & 100 \\
Melezitose & 100 & 0 & 0 & 50 & 0 & 25 & 0 \\
Melibiose & $50^{*}$ & 100 & 33 & 75 & 0 & 100 & 100 \\
Pectin & 100 & 100 & 100 & 100 & 0 & 100 & 100 \\
Raffinose & $100^{*}$ & 100 & 33 & 75 & 0 & 100 & 100 \\
Rhamnose & $75^{*}$ & 0 & 33 & 50 & 0 & 0 & 0 \\
Ribose & 0 & 0 & 0 & 0 & 0 & 0 & 0 \\
Rutin & 100 & 100 & 100 & 100 & 100 & 75 & 100 \\
Salicin & $88^{*}$ & 100 & 100 & 100 & 0 & 100 & 100 \\
Sorbitol & $0^{*}$ & 0 & 0 & 25 & 0 & 0 & 0 \\
Starch & 100 & 100 & 67 & 100 & 100 & 75 & 100 \\
Sucrose & $100^{*}$ & 100 & 100 & 100 & 0 & 100 & 100 \\
Trehalose & $62^{*}$ & 0 & 33 & 50 & 0 & 25 & 0 \\
Xylan & 100 & 100 & 100 & 100 & 100 & 75 & 100 \\
Xylose & $75^{*}$ & 100 & 100 & 75 & 0 & 100 & 100 \\
\hline
\end{tabular}

(Attwood et al., 1996). This may be due to differences in the base medium used, as carbon source utilization was previously assessed in CC medium (Attwood et al., 1996; Leedle \& Hespell, 1980), or it may illustrate plasticity in the expression of these utilization phenotypes. The general ability to use substrates within a single species was variable, as previously observed (Kopečný et al., 2003).

Proteinase activities were assessed by monitoring azocasein degradation by bacterial cells grown in DSM Medium 704 as previously described (Attwood \& Reilly, 1996). Experiments were performed in duplicate. Proteinase activity was observed from all strains examined except $B$. hungatei AR10 and P. ruminis DSM $9787^{\mathrm{T}}$. B. proteoclasticus displayed moderate levels of proteinase activity, along with all strains of B. fibrisolvens, B. hungatei $\mathrm{JK} 615^{\mathrm{T}}$ and DSM 10295, and P. xylanivorans DSM $14809^{\mathrm{T}}$.

VFA production was determined from two replicate broth cultures of each strain grown overnight in DSM Medium 704 and assessed for acetate, propionate, iso-butyrate, $n$ butyrate, iso-valerate and $n$-valerate concentrations on a HP 6890 series GC (Hewlett Packard) with $\alpha$-caproate as the internal standard. All strains tested were net producers of $n$-butyrate, with strain $\mathrm{B} 316^{\mathrm{T}}$ producing the highest concentration, $15.3 \mathrm{mM}$. All strains were net utilizers of the remaining VFAs that were tested for.

Phylogenetic, genetic, morphological and physiological data all support the reclassification of $C$. proteoclasticum to the genus Butyrivibrio where it is genetically and physiologically distinct from extant species of the genus. Hence it is proposed that Clostridium proteoclasticum be reclassified as Butyrivibrio proteoclasticus comb. nov.

\section{Description of Butyrivibrio proteoclasticus comb. nov.}

Butyrivibrio proteoclasticus (pro.te.o.clas'ti.cus. N.L. n. proteinum protein; N.L. adj. clasticus breaking; N.L. adj. proteoclasticus protein breaking).

Basonym: Clostridium proteoclasticum Attwood et al. 1996.

Physiological data and phylogenetic analysis based on $16 \mathrm{~S}$ rRNA gene sequences support the reclassification of Clostridium proteoclasticum as Butyrivibrio proteoclasticus comb. nov. The type strain $\left(\mathrm{B} 316^{\mathrm{T}}\right)$ has the following properties in addition to those given in the original description: growth is supported by dextrin, aesculin, pectin, raffinose, rutin and trehalose, but not by ribose. Converts linoleic acid to stearic acid. The DNA G $+\mathrm{C}$ content of strain $\mathrm{B} 316^{\mathrm{T}}$ is $40.01 \mathrm{~mol} \%$ (as determined from the genome sequence).

The type strain, B316 ${ }^{\mathrm{T}}\left(=\right.$ ATCC $51982^{\mathrm{T}}=$ DSM $\left.14932^{\mathrm{T}}\right)$, was isolated from the rumen of a New Zealand cow fed a fresh, predominantly ryegrass and clover diet.

\section{Acknowledgements}

We thank Graham Naylor for assistance with HPLC analyses and Dr J. Euzéby and Professor H. G. Trüper for expert advice on the Latin nomenclature. This work was funded by the New Economy Research Fund of the Foundation for Research, Science and Technology, New Zealand.

\section{References}

Attwood, G. T. \& Reilly, K. (1995). Identification of proteolytic rumen bacteria isolated from New Zealand cattle. J Appl Bacteriol 79, 22-29.

Attwood, G. T. \& Reilly, K. (1996). Characterization of proteolytic activities of rumen bacterial isolates from forage-fed cattle. J Appl Bacteriol 81, 545-552. 
Attwood, G. T., Reilly, K. \& Patel, B. K. (1996). Clostridium proteoclasticum sp. nov., a novel proteolytic bacterium from the bovine rumen. Int J Syst Bacteriol 46, 753-758.

Bryant, M. P. (1986). Genus IV. Butryrivibrio Bryant and Small 1956, 18, emend. Moore, Johnson and Holdeman 1976, 241. In Bergey's Manual of Systematic Bacteriology, pp. 1376-1379. Edited by P. H. A. Sneath, N. S. Mair, M. E. Sharpe \& J. G. Holt. Baltimore, MD: Williams and Wilkins.

Bryant, M. P. \& Small, N. (1956). The anaerobic monotrichous butyric acid-producing curved rod-shaped bacteria of the rumen. J Bacteriol 72, 16-21.

Cole, J. R., Chai, B., Farris, R. J., Wang, Q., Kulam-Syed-Mohideen, A. S., McGarrell, D. M., Bandela, A. M., Cardenas, E., Garrity, G. M. \& Tiedje, J. M. (2007). The ribosomal database project (RDP-II): introducing myRDP space and quality controlled public data. Nucleic Acids Res 35, D169-D172.

Collins, M. D., Lawson, P. A., Willems, A., Cordoba, J. J., FernandezGarayzabal, J., Garcia, P., Cai, J., Hippe, H. \& Farrow, J. A. (1994). The phylogeny of the genus Clostridium: proposal of five new genera and eleven new species combinations. Int J Syst Bacteriol 44, 812-826.

Felsenstein, J. (1985). Confidence limits on phylogenies: an approach using the bootstrap. Evolution 39, 783-791.

Forster, R. J., Teather, R. M., Gong, J. \& Deng, S. J. (1996). 16S rDNA analysis of Butyrivibrio fibrisolvens: phylogenetic position and relation to butyrate-producing anaerobic bacteria from the rumen of whitetailed deer. Lett Appl Microbiol 23, 218-222.

Kimura, M. (1980). A simple method for estimating evolutionary rates of base substitutions through comparative studies of nucleotide sequences. J Mol Evol 16, 111-120.

Kopečný, J., Marinšek-Logar, R. \& Kobayashi, Y. (2001). Phenotypic and genetic data supporting reclassification of Butyrivibrio fibrisolvens isolates. Folia Microbiol (Praha) 46, 45-48.

Kopečný, J., Zorec, M., Mrázek, J., Kobayashi, Y. \& Marinšek-Logar, R. (2003). Butyrivibrio hungatei sp. nov. and Pseudobutyrivibrio xylanivorans sp. nov., butyrate-producing bacteria from the rumen. Int J Syst Evol Microbiol 53, 201-209.
Leedle, J. A. \& Hespell, R. B. (1980). Differential carbohydrate media and anaerobic replica plating techniques in delineating carbohydrate-utilizing subgroups in rumen bacterial populations. Appl Environ Microbiol 39, 709-719.

Moore, W. E. C., Johnson, J. L. \& Holdeman, L. V. (1976). Emendation of Bacteroidaceae and Butyrivibrio and descriptions of Desulfomonas gen. nov. and ten new species in the genera Desulfomonas, Butyrivibrio, Eubacterium, Clostridium, and Ruminococcus. Int J Syst Bacteriol 26, 238-252.

Mrázek, J. \& Kopečný, J. (2001). Development of competitive PCR for detection of Butyrivibrio fibrisolvens in the rumen. Folia Microbiol (Praha) 46, 63-65.

Paillard, D., McKain, N., Chaudhary, L. C., Walker, N. D., Pizette, F., Koppova, I., McEwan, N. R., Kopecný, J., Vercoe, P. E. \& other authors (2007). Relation between phylogenetic position, lipid metabolism and butyrate production by different Butyrivibrio-like bacteria from the rumen. Antonie van Leeuwenhoek 91, 417-422.

Saitou, N. \& Nei, M. (1987). The neighbor-joining method: a new method for reconstructing phylogenetic trees. Mol Biol Evol 4, 406-425.

Stackebrandt, E. \& Goebel, B. M. (1994). Taxonomic note: a place for DNA-DNA reassociation and $16 \mathrm{~S}$ rRNA sequence analysis in the present species definition in bacteriology. Int J Syst Bacteriol 44, 846-849.

Staley, J. T. (2006). The bacterial species dilemma and the genomicphylogenetic species concept. Philos Trans R Soc Lond B Biol Sci 361, 1899-1909.

Tamura, K., Dudley, J., Nei, M. \& Kumar, S. (2007). MEGA4: molecular evolutionary genetics analysis (MEGA) software version 4.0. Mol Biol Evol 24, 1596-1599.

Wallace, R. J., Chaudhary, L. C., McKain, N., McEwan, N. R., Richardson, A. J., Vercoe, P. E., Walker, N. D. \& Paillard, D. (2006). Clostridium proteoclasticum: a ruminal bacterium that forms stearic acid from linoleic acid. FEMS Microbiol Lett 265, 195-201.

Willems, A., Amat-Marco, M. \& Collins, M. D. (1996). Phylogenetic analysis of Butyrivibrio strains reveals three distinct groups of species within the Clostridium subphylum of the gram-positive bacteria. Int $J$ Syst Bacteriol 46, 195-199. 